\title{
Reconstruyendo la materia de Didáctica de la Educación Física desde la perspectiva autobiográfica del alumnado Reconstructing the subject of Didactics of Physical Education from the autobiographical perspective of the teaching student Raúl Eirín Nemiña \\ Universidad de Santiago de Compostela (España)
}

Resumen. El propósito de este artículo es el de investigar las experiencias y recuerdos de las clases de Educación Física (EF) que tienen los alumnos y alumnas de la materia de Didáctica de la EF del grado de de maestro de la USC. Se ha hecho analizando el discurso que presentan los estudiantes de la materia, apoyándose en la teoría de la memoria autobiográfica, mediante textos autobiográficos de sus recuerdos de la EF escolar. Los participantes han sido 60 alumnos (47 mujeres y 13 hombres) de la materia Didáctica de la EF, de la titulación de Grado en Maestro de la USC. Para acceder a sus autobiografías se han usado relatos elaborados de sus recuerdos escolares. El análisis de los datos se ha usado desde un enfoque de indagación naturalista (Lincoln \& Guba, 1985) a través de la lectura reiterada de los textos elaborados por los alumnos, identificando temas recurrentes y patrones que dan como resultado el establecimiento de categorías provisionales que, una vez decantadas, conforman los temas centrales y el contenido del discurso que expresan los textos. Estos se agrupan en torno a recuerdos agradables y positivos, memorias negativas e influencias del profesor. Estas concepciones que traen los futuros docentes sirven como contexto para confrontarlos con una idea de EF escolar que rompa estos estereotipos. Apoyándonos en una idea de EF inclusiva e integradora, favorecemos la adquisición de un espíritu crítico para que, como futuros docentes de educación primaria, construyan sus propios significados de la EF en la escuela y en su identidad profesional.

Palabras clave. Didáctica de la Educación Física, formación inicial de profesores, autobiografías, investigación narrativa, recuerdos como alumno.

\begin{abstract}
The purpose of this article is to investigate the experiences and memories of Physical Education classes (PE) that have the students of the subject of Didactics of PE of the degree of Primary Education of the USC. It has been done analyzing the discourse presented by the students of the subject, relying on the theory of autobiographical memory, through autobiographical texts of their memories of school PE. The participants were 60 students (47 women and 13 men) of the subject Didactic of PE. To access his autobiographies, elaborate accounts of his school memories have been used. The analysis of the data has been used from a naturalistic inquiry approach (Lincoln \& Guba, 1985) through the repeated reading of the texts elaborated by the students, identifying recurring themes and patterns that result in the establishment of provisional categories that once settled, they form the central themes and content of the discourse expressed in the texts. These are grouped around pleasant and positive memories, negative memories and influences of the teacher. These conceptions that the future teachers bring serve as a context to confront them with an idea of school PE that breaks these stereotypes. Based on an inclusive and integrative idea of PE, we favour the acquisition of a critical spirit so that, as future teachers of primary education, they construct their own meanings of PE in the school and in their professional identity.
\end{abstract}

Keywords. Physical education, teacher's initial training, autobiographies, narrative research, memories.

\section{Introducción}

Las concepciones y creencias sobre la escuela elaboradas en los primeros momentos de la vida de las personas influyen en la asimilación de los contenidos propios de la formación inicial como profesores. Este sistema de creencias que «traen» los futuros profesores sirve como lente a través de la cual contemplan los programas de formación. Las concepciones sobre la enseñanza influyen en el desempeño docente, por lo que parece oportuno estudiar cómo afectan a la formación de profesores y la calidad de la educación que estos proporcionan (Impedovo, 2016). Es por tanto importante que, como responsables de los programas de formación de los futuros docentes, tengamos en cuenta los conocimientos previos y creencias que traen nuestros alumnos, ya que las nuevas ideas y planteamientos tienen que competir con las creencias y teorías elaboradas de forma tácita e implícita en su propia historia como alumnos, a lo largo de su experiencia como alumnos en el sistema educativo.

La perspectiva constructivista del aprendizaje (Borko, 2004; Darling-Hammond \& McLaughlin, 2011; Le Fevre \&

\footnotetext{
Fecha recepción: 12-02-19. Fecha de aceptación: 08-09-19 Raúl Eirín-Nemiña
} raul.eirin@usc.es
Richardson, 2002) supone que la tarea del educador consiste en proporcionar oportunidades e incentivos para que los alumnos construyan su propio conocimiento. Desde esta perspectiva se ve el aprendizaje como un proceso de construcción no lineal, sino interpretativo y recursivo donde los aprendices interactúan con el conocimiento a trabajar y el contexto físico y social en el que están integrados. Se concibe más como una actividad que desarrollan los aprendices y se hacen a sí mismos, en vez de algo que «adquieren» de otros. Es importante, por tanto, intentar comprender como los futuros docentes interpretan, piensan y sienten el contenido que trabajan, así como las concepciones que traen y como estas afectan a la interpretación de su contexto de enseñanza.

Varias investigaciones han señalado que las experiencias previas como alumno de EF influyen en las actitudes, creencias y prácticas de los futuros profesores (Lawson, 1986; Belka, Lawson \& Cross-Lipnickey (1991); Doolittle, Dodds \& Placek (1993); Placek, Dodds, Doolittle, Portman, Ratliffe \& Pinkham (1995); Keating, Silverman \& Kulinna (2002). Allison, Pissanos \& Sakola (1990) nos hablan de los recuerdos positivos y negativos de sus alumnos respecto a las clases de EF, otorgándole un papel destacado a sus antiguos profesores en las concepciones e ideas sobre la EF. 
También Portman (1996) trabaja sobre los recuerdos de sus alumnos, futuros profesores, destacando las influencias que refieren de sus buenas experiencias escolares, así como la voluntad de no repetir sus malas experiencias y situaciones desagradables. La teoría de la socialización docente describe la construcción de la identidad profesional como un proceso que comienza en la escuela como alumno y que se extiende a lo largo de toda la vida del docente. Crum (1993) nos recuerda que los docentes de EF no llegan a la formación inicial como «tabula rasa», sino que traen consigo concepciones y experiencias entresacadas de sus clases de EF y la participación en actividades recreativas y deportivas fuera del contexto escolar.

Los estudiantes que acceden a los programas de formación inicial de profesores suelen ingresar con preconcepciones arraigadas sobre la enseñanza y el aprendizaje, de las que rara vez son conscientes (McDiarmid 1990). Están basadas en los años de experiencia educativa como estudiantes, lo que se ha denominado «aprendizaje por observación» (Feiman-Nemser \& Remillard 1996; Lortie 1975; Sugrue 1996). Este conocimiento previo sobre la enseñanza actúa, en palabras de Bullough \& Gitlin (2001), como un filtro o lente a través del cual los docentes en formación piensan, actúan y por tanto configura su práctica docente. En general, los programas de formación de profesores diseñan experiencias que ayudan a los futuros profesores a explicitar sus ideas preconcebidas y a examinar críticamente sus experiencias. Sabemos que los nuevos conocimientos presentados en la formación docente que contradicen las creencias sostenidas tienden a ser descartados o ignorados (Bullough \& Gitlin, 2001), debido a la potencia de estas creencias iniciales sobre la enseñanza y el aprendizaje. La investigación ha señalado reiteradamente que los programas de formación docente no cambian las creencias que traen los futuros docentes y que las prácticas que experimentaron como exitosas cuando eran estudiantes, son aquellas a las que recurren una vez comienzan su ejercicio profesional. Como nos recuerda Calderhead (1996), los futuros docentes no suelen cambian sus concepciones, a menos que enfrenten y cuestionen sus creencias a través de experiencias significativas que les hagan reconocer y valorar los procesos de cambio, tanto para ellos mismos como para sus alumnos.

Parece oportuno que los programas de formación docente comprendan las experiencias, creencias y teorías implícitas que «traen» los futuros profesores. Esto permite confrontarlas, ayudarles a reconocerlas, y a reflexionar críticamente para fomentar un cambio que pueda impactar en su práctica docente (Calderhead 1996; O’Sullivan, 2005; Pajares 1992; Tsangaridou \& O’Sullivan, 2003). La docencia es el resultado de la acumulación de conocimientos, creencias, experiencias, sensaciones y sentimientos recogidos a lo largo de su vida personal y profesional. Examinar los relatos de los futuros profesores ayuda a reconocer sus creencias preexistentes sobre la enseñanza y el aprendizaje, les invita a reflexionar y repensar estas creencias y a ver cómo afectan a sus actuaciones con los alumnos. A través del lenguaje, las expresiones y metáforas que utilizan, trasladan sus ideas y concepciones sobre lo que significa enseñar, lo que en cierto modo guía su acción docente.

El acercamiento a los relatos y recuerdos como alumnos, ayuda a comprender y situar -cuando son analizados críticamente- la historia, evolución, situación actual y perspectivas de futuro de la EF en la escuela. Este conjunto de ideas, valores, concepciones, normativas y rutinas orientan la práctica profesional. Nuestro trabajo como responsables de transmitir y ayudar a configurar la idea de EF a los futuros maestros es precisamente este. Ayudarles a que configuren en su identidad profesional una idea de la EF desde una perspectiva educativa, que atienda a los ámbitos físico, cognitivo y afectivo de los alumnos, hacerlos conscientes de su importancia y utilidad en la escuela.

Este acercamiento a la metodología autobiográfica en la formación inicial de profesores se ha utilizado, entre otros, para estudiar los recuerdos de las emociones hacia las materias de ciencias y matemáticas en secundaria, por parte de los alumnos del máster en educación secundaria (Costillo, Borrachero, Brígido, \& Mellado (2013). También se trabaja sobre los recuerdos de los alumnos del grado de Primaria, sobre la consistencia metodológica que han recibido en la enseñanza de la Historia (Gómez, Rodríguez \& Mirete (2016), la construcción de la identidad profesional y la formación permanente de profesores de EF (González Calvo \& Barba, 2014), la perspectiva autobiográfica de los docentes noveles sobre los aprendizajes de la EF (González-Calvo, G.; Barba, J. J. (2013); los diarios corporales como acercamiento al prácticum de la titulación de maestro (González-Calvo, \& Martínez Álvarez, 2018); la idea que reflejan sobre la construcción social de la EF por parte de los futuros maestros Valencia-Peris \& Lizandra (2018). También Beltrán-Carrillo \& Devís-Devís (2019) estudiando el pensamiento de los alumnos en EF recurren a sus recuerdos, al igual que Sinelnikov \& Hastie (2010) trabajando sobre el modelo de enseñanza de la EF denominado educación deportiva y Morgan \& Bourke (2008) comparando las actuaciones docentes de maestros generalistas y especialistas de EF.

Para ello, y siguiendo las recomendaciones de partir de los conocimientos previos de los alumnos, adoptamos la estrategia de proponer un trabajo de recuerdo de sus experiencias y sensaciones de la EF en su etapa escolar. Este trabajo nos permite introducirnos en dos elementos clave en la formación de los futuros docentes. En primer lugar, permite un contexto próximo y propio que aportan los alumnos, y en segundo lugar permite a los alumnos una reflexión y análisis de sus propias creencias y significados de los que es la EF en la escuela. En este documento presentamos algunos hallazgos extraídos de estos comentarios y concluimos con la idea de validez y oportunidad de esta forma de trabajo para acercarnos a las concepciones sobre la EF que traen, a la formación inicial, los futuros docentes.

\section{Metodología}

En la materia de Didáctica de la EF se mantiene un trabajo de recogida de «recuerdos y experiencias como alumnos de EF» que sirve como plataforma para introducir la asignatura y conectar con los intereses y experiencias de los alumnosfuturos docentes. Se plantea al inicio de la docencia de la materia, y se desarrolla durante las primeras semanas de contacto con los estudiantes. A través de esta autonarrativa que realizan los propios alumnos, se construye una pequeña his- 
toria de vida de sus experiencias en la EF escolar.

\section{Recogida de la información}

Los participantes son 60 estudiantes (47 mujeres y 13 hombres) de la materia Didáctica de la EF; se imparte en la titulación de Grado en Maestro de Educación Primaria, ubicada en el segundo cuatrimestre del segundo año. Se les propone a los alumnos una reflexión sobre sus concepciones y creencias acerca de la enseñanza y el aprendizaje de la EF en la escuela. A través de la escritura, el análisis y la revisión de sus recuerdos, regresan a sus experiencias como aprendices en el entorno escolar. Para ello, de forma individual, tienen que elaborar un relato autobiográfico sobre sus experiencias como alumno de EF en su etapa escolar, previa a la entrada a la universidad. Si bien se centra en la etapa educativa, pueden incluir apreciaciones sobre la participación en actividades deportivas durante los años escolares. Se establece una extensión aproximada de 1000 palabras. Se ha solicitado permiso a los alumnos para usar sus reflexiones para trabajos de investigación. Para garantizar la confidencialidad de las opiniones de los participantes, en este trabajo hemos recurrido al uso de pseudónimos al introducir las voces de los alumnos en el texto.

\section{Análisis de datos}

Dado el carácter cualitativo del estudio, se utilizó un enfoque de indagación naturalista (Lincoln \& Guba 1985). A través de lectura repetida de los relatos, se han ido identificando temas y patrones recurrentes que se han establecido como categorías provisionales. De forma inductiva (Patton, 2001), mediante un proceso de decantación de los significados atribuidos en los textos, se han establecido las categorías definitivas. Para asegurar la validez y fiabilidad del proceso hemos contado con el contraste y discusión con otro investigador, así como las aclaraciones proporcionadas por los participantes para aclarar o matizar los textos elaborados. Sobre la base de este proceso, los datos se organizaron en torno a tres categorías, «buenos recuerdos»- experiencias positivas; «para olvidar»- experiencias desagradables y «profesorado».

\section{Resultados y discusión}

En este apartado mostramos extractos del discurso de los alumnos que apoyan la elaboración de las categorías. Para ello elaboramos una secuencia en la que se intercalan las categorías elaboradas, con los extractos del discurso que las apoyan. También aportamos la discusión de los hallazgos con aquellos recogidos en la literatura.

\section{«Buenos recuerdos», experiencias positivas de la asig- natura}

En general la mayoría de los relatos se muestran como positivos, buenos, agradables y entusiastas. La mayor parte de los relatos identifican las clases de EF como un tiempo de juego, diversión, pasarlo bien, dinamismo. Se asocia a tiempo de ocio y libertad, como contrapeso a la rigidez de las demás materias escolares que se realizan en el aula.

«...para mi y mis compañeros era tiempo para divertirnos, para desconectar y liberar energía...» (Carmen). «...me encantaba llegar a la clase de EF, pues el profe preparaba unas clases muy dinámicas y divertidas en las que hacíamos circuitos, juegos y distintos deportes « (María).

«...es una materia que nos gustaba, pues salías de la rutina de estar sentado en el aula» (Andrea).

«...es como la hora del recreo, donde te relajas y desconectas del agobio de las demás materias» (Paula).

«...esos días estábamos más contentos de lo habitual, pues es como si hubiese dos recreos...» (Clara).

«...sinónimo de tiempo de desconexión del pupitre el encerado y los libros» (Alfonso).

También aparecen alusiones positivas al trabajo con los demás compañeros y la relación un poco especial que se establece con el profesor de EF, alejada del formalismo que se mantiene en el aula tradicional

«...trabajar con los compañeros y realizar actividades divertidas que nos sacaban de la rutina de los libros de texto...» (Alba).

«...me gustaba porque podías correr, hablar, divertirte, interrelacionar con tus compañeros, y la relación con el profesor era distinta...»(Teresa).

En definitiva, las buenas valoraciones de los recuerdos hacia la materia se resumen en la expresión de Ángel cuando nos dice

«...siempre me gustó esta materia, la que más; era una liberación para nosotros a esa edad poder jugar y aprender...» (Ángel).

Estas apreciaciones positivas, cercanas a la función catárquica y hedonista de la EF, coinciden con otros trabajos sobre el interés de los alumnos hacia la EF, por ejemplo Moreno \& Hellín (2007) sobre los alumnos de secundaria, o Valencia-Peris \& Lizandra (2018) con futuros profesores de primaria. También Kretchmar (2006), cuando nos recuerda que las razones para una EF escolar de calidad, además de la argumentación sobre la salud, la necesaria competencia motriz y la concepción educativa integral de la persona, la EF escolar tiene que ser juego y diversión, significativa y parte de la identidad personal para contribuir a la libertad para crear, expresar y descubrir. Tiene que ayudar a hacer mejor la vida de nuestros alumnos, no sólo más larga.

\section{«Para olvidar»- experiencias desagradables}

En menor medida numérica, aunque no con menor importancia, aparecen apreciaciones negativas, desagradables, pesimistas, con situaciones problemáticas y malas experiencias. Como señala María, en su relato aparece la EF como

«...lugar de soledad, aislamiento y desgana...» (María L.B.).

Los motivos que llevan a estas situaciones son variados; algunos tienen que ver con una baja competencia motriz, sostenida en el tiempo, y sin posibilidad de abrirse a otras posibilidades por un currículo demasiado estrecho, centrado en la práctica deportiva

«...no tengo buenos recuerdos de las clases de EF, pues nunca se me dieron bien los deportes, y era lo único que practicábamos» (Noemí C.B.).

También aparece un decreciente interés hacia la materia, siendo en general muy alto en la educación infantil y primaria, con un acusado descenso en la secundaria y el bachille- 
rato. Mientras el componente lúdico y participativo «abunda» en los relatos sobre las etapas de infantil y primaria, como ya hemos señalado, en la etapa de secundaria aparecen con fuerza los contenidos deportivos y de condición física que, en un enfoque técnico-tradicional, van dejando que aparezcan comentarios negativos en el sentimiento de pertenencia y participación en la clase,

«...si un niño no era habilidoso en ningún deporte, era marginado por el resto de los alumnos e incluso por el profesor» (Andrea L.L.)

Las apreciaciones vertidas por los alumnos respecto a los materiales e instalaciones ofrecen una visión de la situación de valor otorgado a la materia de EF en el contexto en el que fueron escolarizados. Así en general se habla del patio, instalaciones deportivas (pabellones de deportes) y gimnasios. Suele ser habitual que la instalación deportiva (pabellón) tenga mayor valoración y por tanto un uso más exclusivo para aquellos centrados en los deportes de exhibición, mientras que las clases de EF (más humildes) se desarrollen en el patio, gimnasios, o salas habilitadas para tal fin con material, con material viejo, gastado y sucio

«....nosotros nos quedábamos en una esquina donde estaba todo sucio roto, con balones viejos, mesas y sillas rotas...» (Rebeca)

También aparecen varios comentarios negativos referidos a la evaluación

«...la diferencia en la nota radicaba en la condición física de los alumnos (resistencia, velocidad, flexibilidad,...) así como si practicaban deporte fuera de la escuela». (Lucía).

«...el tiempo de aseo posterior era obligatorio, con faltas si te olvidabas de la camiseta, la toalla o el chandal obligatorio impuesto por el colegio...) (Andrea L.O.).

«el profesor establecía una calificación más baja a los alumnos que no participaban en las actividades deportivas del colegio, tratando de «reclutar» al alumnado» (Tania J.C.);

«a la hora de calificar, el docente puntuaba a los niños con sobresaliente, mientras que las niñas aspiraban a tener como nota máxima un 7» (Andrea L.O.).

Este último comentario nos trae las experiencias vividas por los alumnos discriminados por razón de género,

«...en general, el profesor de Educación Física solía prestar más atención a los niños que las niñas, y asociaba cierto tipo de juegos, como es el fútbol o el baloncesto, al género masculino, dejándonos relegadas en un área muy pequeña del pabellón, y jugando a juegos que se consideraban femeninos como es la comba o la mariola» (Paula D.M.)

Aparte de la razón de género, los alumnos se sienten apartados y con menor consideración cuando presentan problemas de obesidad, sedentarismo y en general baja competencia motriz. Especialmente en modelos docentes tradicionales que enfatizan las habilidades técnicas, el desarrollo de las capacidades físicas básicas y un ideal de modelo corporal dominante, los alumnos que no se ajustan a este patrón padecen una doble discriminación, por parte de compañeros y el profesor,

«...llegábamos a fingir lesiones para escaquearnos de la sesión...») (Andrea L.L.); «...muchos de los juegos se basaban en la competitividad y en la presión ejercida por ciertos compañeros que, si no eras muy habilidosos, solían gritarte,» (Tania J.C.).

«...como fueras «el gordito», siempre te dejan al final a la hora de hacer los equipos...» (Joaquina)

Sin llegar a las situaciones de miedo, descritas por Monforte \& Pérez-Samaniego (2017), parece claro que no todas las experiencias y recuerdos son agradables. La búsqueda de un currículo de educación física inclusivo en el que no se destaque la baja competencia motriz de los alumnos, cuidar una evaluación formativa, centrada en el proceso y la mejora de los valores educativos, pensamos que pueden ser estrategias que ayuden a superar estos desencantos.

\section{La labor del profesor}

La visión de la labor del profesor, como elemento medular de las clases de EF, ayuda a conformar la idea de lo que es la educación física. En cierto modo aparece como la «representación» de la materia. En este sentido aparece como causante de buenos recuerdos y experiencias

«...tengo buenos recuerdos del profe, era agradable y sonriente, y se notaba que le gustaba dar clase...» (Antonio);

«...los docentes de primaria solían mostrar mayor satisfacción en las sesiones...» (Beatriz P.B.)

Por otra parte, aparecen elementos de crítica y escasa valoración, relacionados a veces con su escasa profesionalidad

«...el profesor, con un enfoque tradicional, se limitaba al juego libre, incurriendo en una cierta negligencia al no tener en cuenta las recomendaciones del currículo y limitarse a disminuir su carga de trabajo» (María C.B.).

Los estudiantes señalan como elementos negativos de sus profesores, aquellas concepciones demasiado escoradas hacia la vertiente deportiva-competitiva, lo que provoca en sus alumnos sentimientos de desencanto y falta de implicación en la materia

«...si un niño no era habilidoso en ningún deporte, era marginado por el resto de los alumnos e incluso por el profesor» (Andrea L.L.)

También destacan las características del profesor que no concuerdan con la idea general del comportamiento en clases de educación física

«...mi primer profesor (generalista) no me gustaba mucho porque, entre otras cosas, fumaba habitualmente mientras impartía clase» (Virginia).

Más allá de la anécdota del profesor fumador, esta cita trae el tema de la EF física impartida por profesores generalistas-especialistas. En general, dada la edad de nuestros alumnos, la gran mayoría han tenido profesores especialistas y solo aquellos alumnos de mayor edad recuerdan las clases impartidas por el profesor generalista que, e muchos casos, asocian a tiempo de juego libre o prolongación del recreo.

Para terminar, podemos decir que la educación física genera una mezcla de sentimientos. Por un lado es una materia que suele gustar por desenvolverse en un contexto diferente (patio, gimnasio,...), alejada de las disposiciones y convenciones más académicas de la escuela, con unas actitudes 
y comportamientos relajados y cercanos. Se trabaja con el propio cuerpo, con compañeros y con materiales cercanos de las actividades lúdicas, desarrollando conocimientos, actitudes y valores. Por otra parte, los elementos curriculares y relacionales están profundamente influenciados por la figura del docente, lo que genera una dependencia destacada de esta figura que, en un tono positivo atrae, apoya y estimula, o por el contrario genera sentimientos encontrados y poco gratificantes de exclusión y temor, a veces pánico, especialmente entre aquellos alumnos que se sienten discriminados por cuestiones de género o habilidad motriz.

\section{Implicaciones prácticas de la experiencia}

Las experiencias personales que han tenido los docentes en su etapa como alumnos, son componentes importantes de su identidad como educadores. Al trabajar con ellas ponemos de relieve la teoría del aprendizaje social de Bandura $(1977,1986)$ en la que se asume que la habilidad de una persona para gestionar una determinada situación se apoya en sus experiencias previas. Pensamos que el trabajo con narrativas autobiográficas facilita la integración de conocimientos y experiencias, posibilitando la reflexión sobre los elementos teórico-prácticos que componen el programa de la materia de Didáctica de la EF. Por tanto, ayuda a los futuros docentes a repensar sus creencias y asunciones sobre la EF, al tiempo que favorece la conformación de su identidad profesional, ampliando sus conocimientos y concepciones de lo que es la EF escolar.

En este estudio cualitativo hemos analizado los recuerdos y experiencias con la EF que han tenido, en su etapa escolar, un grupo de estudiantes del grado de maestro de la USC. Se ha propuesto, dentro de las actividades a realizar en la materia de Didáctica de la EF, la elaboración de relatos autobiográficos sobre sus experiencias como alumnos de EF en la etapa educativa y actividad deportiva escolar. Estos textos, de alrededor de mil palabras han sido leídos, compartidos y categorizados por los alumnos en grupos pequeños, y por el docente encargado de la materia. De este trabajo reflexivo y colaborativo se han ido desprendiendo las categorías de análisis que se presentan en los resultados. Es de destacar, como «efectos colaterales» de esta metodología, el influjo de las experiencias personales como alumnos, en el desarrollo de actitudes y conocimientos hacia la materia por parte de los futuros docentes. Si bien inicialmente los alumnos se muestran un tanto reacios y escépticos, con textos en algunos casos descriptivos y anecdóticos, también aparecen relatos con mayor profundidad en los que se describen sentimientos y situaciones con gran potencial educativo. El trabajo en pequeño grupo de análisis y categorización, refuerza esta posibilidad de uso educativo de los relatos. La confrontación de ideas y perspectivas que aparecen en los textos, así como los debates que se mantienen en clase, ayudan a ampliar las concepciones del sentido de la EF en la escuela que traen los futuros profesores. También favorecen y complementan las perspectivas históricas que han configurado la EF escolar, al tiempo que estudian e interiorizan el actual currículo de EF.

Del análisis de sus discursos extraemos tres categorías que dominan sus textos, buenas experiencias, recuerdos desagradables y profesorado. Los datos sugieren que, en general, mantienen recuerdos agradables y positivos de sus clases de EF, siendo los aspectos que valoran en mayor medida el ambiente informal y distendido, las posibilidades de relación e interacción con los compañeros y el profesor, así como los espacios y materiales cercanos a sus propuestas lúdicas. También aparecen experiencias negativas, agrupadas en torno a situaciones de discriminación por motivos de género, habilidad motriz, identidad corporal y evaluaciones de la materia (estrechas) centradas en el rendimiento y habilidad.

Estas situaciones y vivencias afectan negativamente a sus comprensiones de lo que significa la EF escolar, que arrastran durante su formación inicial y, si no son tratadas, acaban reproduciendo en su ejercicio profesional. En este sentido, entendemos que es importante que los formadores de los futuros docentes tengan en cuenta estas vivencias, $\mathrm{y}$ las utilicen para fomentar una idea de la educación física alejada de los valores tradicionales y hegemónicos y más próxima a los valores inclusivos, participativos y democráticos que deberían guiar la EF escolar. Como apoyo a esta labor de construcción de una idea educativa de la EF escolar, es importante que los futuros docentes tengan una visión crítica de la EF, la actividad física y el deporte. En este sentido, identificando situaciones negativas apoyadas en sus propias experiencias y en los discursos sociales dominantes, analizando las consecuencias y proponiendo soluciones alternativas, nos ha servido para profundizar en sus comprensiones de la materia y ampliar sus concepciones y expectativas con la asignatura.

Si bien en este artículo nos hemos centrado en las tres categorías de análisis presentadas, nos parece interesante destacar las posibilidades de esta metodología como medio para situar problemas y dilemas de la materia de Didáctica de la EF. El influjo que ejercen las experiencias personales como alumnos en EF, juegan un papel importante en el desarrollo de actitudes y conocimientos hacia la materia por parte de los futuros docentes. Partiendo de las experiencias vividas y sentidas de los alumnos, se puede ampliar el campo de visión de la EF que tienen los futuros profesores. Esta reflexión profunda hace que se cuestionen discursos hegemónicos y prácticas instauradas en la EF que necesitan ser confrontadas y sustituidas por un enfoque inclusivo y de respeto hacia todo el alumnado independientemente de sus características individuales.

Para finalizar, entendemos que el profesorado es el agente más influyente en esta situación, por lo que se requiere de su ejemplo de fomento de la inclusión, velando por aquellos alumnos que sufren cualquier tipo de discriminación o marginación y enfrentándose a los discursos que la promueven, y favoreciendo la construcción de identidades positivas. En definitiva, evitando situaciones en que se vive la EF como «...lugar de soledad, aislamiento y desgana...» $\mathrm{y}$ convertirla, en palabras de Kretchmar (2006), en un instrumento para hacer mejores las vidas de nuestros alumnos.

\section{Referencias}

Allison, P. C., Pissanos, B. W. \& Sakola, S. P. (1990). Physical education revisited: The institutional biographies of preservice classroom teachers, Journal of Physical Education, Recreation and Dance, 61(5), 76 - 79. 
Bandura, A. (1977). Self-efficacy: toward a unifying theory of behavioral change, Psychological Review, 84(2), 191-215.

Bandura, A. (1986). Social foundations of thought and action: a social cognitive theory (Englewood Cliffs, NJ, Prentice-Hall).

Belka, D. E., Lawson, H. A. \& Cross-Lipnickey, S. C. (1991). An exploratory study of undergraduate recruitment into several major programs at one university, Journal of Teaching in Physical Education, 10(3), 286 - 306.

Beltrán-Carrillo, V. J. \& Devís-Devís, J. (2019). El pensamiento del alumnado inactivo sobre sus experiencias negativas en educación física: los discursos del rendimiento, salutismo y masculinidad hegemónica. RICYDE. Revista internacional de ciencias del deporte. 55(15), 20-34. https://doi.org/10.5232/ ricyde2019.05502.

Borko, H. (2004). Professional development and teacher learning: Mapping the terrain. Educational Researcher, vol. 33(8), 3-15. https://doi.org/10.3102/0013189X033008003

Bullough, R.V. \& Gitlin, A. (2001). Becoming a student of teaching: Methodologies for exploring self and school context. 2nd ed. Madison: Brown and Benchmark.

Calderhead, J. (1996). Teachers: Beliefs and knowledge. En D. C. Berliner y R. C. Calfee (Eds.), Handbook of educational psychology (pp. 709-725). New York, NY, US: Macmillan Library Reference Usa; London, England: Prentice Hall International.

Costillo Borrego, E., Borrachero Cortés, A., Brígido Mero, M. \& Mellado Jiménez, V. (2013). Las emociones sobre la enseñanza-aprendizaje de las ciencias y las matemáticas de futuros profesores de Secundaria. Revista Eureka sobre Enseñanza y Divulgación de las Ciencias, 10, 514-532.

Crum, B. J. (1993). Conventional thought and practice in physical education: problems of teaching and implications for change, Quest, 45, 339 - 356.

Darling-Hammond, L., \& McLaughlin, M. W. (2011). Policies that support professional development in an era of reform; policies must keep pace with new ideas about what, when, and how teachers learn and must focus on developing schools' and teachers' capacities to be responsible for student learning. Phi Delta Kappa, vol. 92 (6), 81-102.https://doi.org/10.1177/ 003172171109200622

Doolittle, S. A., Dodds, P. \& Placek, J. H. (1993). Persistence of beliefs about teaching during formal training of preservice teachers, Journal of Teaching in Physical Education, 12(4), 355- 365.

Feiman-Nemser, S., \& Remillard, J. (1996). Perspectives on learning to teach. En The teacher educator's handbook: Building a knowledge base for the perception of teachers, Murray F.B. (Edt.) 63 - 91. San Francisco: Jossey Bass.

Gómez, C. J., Rodríguez, R. A., \& Mirete, A. B. (2016). Relación entre metodología docente y uso de recursos de innovación en la enseñanza de la historia. Un análisis a través de los recuerdos de los maestros en formación. Clío. History and History Teaching, 42. Recuperado de http://clio.rediris.es

González-Calvo, G. y Barba, J. J. (2013). «Perspectiva autobiográfica de un docente novel sobre los aprendizajes de Educación Física en diferentes niveles educativos en educación física». Cultura, Ciencia y Deporte, 24, 171-181.

González Calvo, G. y Barba, J. J. (2014). Formación permanente y desarrollo de la identidad reflexiva del profesorado desde las perspectivas grupal e individual. Profesorado. Revista de $\mathrm{Cu}$ rrículum y Formación del Profesorado, 18(3), 397-412. Recuperado de http://www.ugr.es/local/recfpro/rev181COL12.pdf

González Calvo, G., \& Martínez Álvarez, L. (2018). Los Diarios Corporales Docentes como Instrumentos de Reflexión y de Evaluación Formativa en el Prácticum de Formación Inicial del Profesorado. Estudios PedagóGicos, 44(2), 185-204. doi:10.4067/S0718-07052018000200185
Impedovo, M. (2016). In-service Teachers' Sense of Agency after Participation in a Research Master Course. International Journal of Educational Psychology, 5(3), 281-307. doi:http://dx.doi.org/ 10.17583/ijep.2016.2206.

Keating, X. D., Silverman, S. \& Kulinna, P. H. (2002). Preservice physical education teacher attitudes toward fitness tests and the factors influencing their attitudes, Journal of Teaching in Physical Education, 21(2), 193 - 207.

Kretchmar, R.S. (2006). Ten More Reasons for Quality Physical Education, Journal of Physical Education, Recreation \& Dance, 77:9, 6-9. DOI: 10.1080/07303084.2006.10597932

Lawson, H. A. (1986). Occupational socialization and the design of teacher education programs, Journal of Teaching in Physical Education, 5(2), 107 - 116.

Le Fevre, D., \& Richardson, V. (2002). Staff development in early reading intervention programs: The facilitator. Teaching and Teacher Education, vol. 18(4), 483-500. https://doi.org/10.1016/ S0742-051X(02)00011-2

Lincoln, Y., \& Guba, E. (1985). Naturalistic inquiry. New York: Sage.

Lortie, D. (1975). Schoolteacher: A sociological study. Chicago: UCP.

McDiarmid, G.W. (1990). Challenging prospective teachers’ beliefs during early ûeld experiences: A quixotic undertaking? Journal of Teacher Education 41, no. 3: 12 - 20.

Monforte, J., \& Pérez-Samaniego, V. (2017). El miedo en Educación Física: una historia reconocible. Movimento, 23(1), 85100

Moreno, J. A. \& Hellín, M. G. (2007). El interés del alumnado de Educación Secundaria Obligatoria hacia la Educación Física. Revista Electrónica de Investigación Educativa, 9(2), 1-20.

Morgan, P., \& Bourke, S. (2008). Non-specialist teachers' confidence to teach PE: The nature and influence of personal school experiences in PE. Physical Education and Sport Pedagogy, $13,1-29$.

O’Sullivan, M. (2005). Learning to teach physical education. In Student learning in physical education: Applying research to enhance instruction, ed. S. Silverman and C. Ennis, 315 - 37. Champaign, IL: Human Kinetics.

Pajares, M.F. (1992). Teachers' beliefs and educational research: Cleaning up a messy construct. Review of Educational Research. 62 (3), $307-32$.

Patton, M. Q. (2001). Qualitative research and evaluation and methods (3rd edn) (Beverly Hills, Sage)

Placek, J. H., Dodds, P., Doolittle, S. A., Portman, P. A., Ratliffe, T. A. \& Pinkham, K. M. (1995). Teaching recruits' physical education backgrounds and beliefs about purposes for their subject matter, Journal of Teaching in Physical Education, 14(3), $246-261$.

Portman, P. A. (1996). Preservice elementary education majors beliefs about their elementary phys- ical education classes (Pt. 1), Indiana Association for Health, Physical Education, Recreation and Dance Journal, 25(2), 25 - 28.

Sinelnikov, O. A., \& Hastie, P. A. (2010). Students' autobiographical memory of participation in multiple sport education seasons. Journal of Teaching in Physical Education, 29, 167 - 183.

Sugrue, C. (1996). Student teacher lay theories: Implications for professional development. En Teachers' professional lives, Goodson, I. y Hargreaves, A. (Eds.) 154 - 77. London: Falmer Press.

Tsangaridou, N. \& O’Sullivan, M. (2003). Physical Education Teachers' Theories of Action and Theories-In-Use. Journal of Teaching in Physical Education, 22:2, 132-152.

Valencia-Peris A. \& Lizandra, J. (2018). Cambios en la representación social de la educación física en la formación inicial del profesorado. Retos. Nuevas Tendencias en Educación Física, Deporte y Recreación, 34, 230-235. 